\section{$\Rightarrow$ HYPERTENSION}

\section{Community-based plan lowers CVD risk}

A tailored, community-based intervention led by non-physician health workers (NPHWs) in two middle-income countries substantially reduces hypertension and the risk of cardiovascular disease (CVD). These outcomes from the HOPE 4 programme were presented at the ESC Congress 2019.

Despite the availability of effective and inexpensive drugs that target CVD risk factors, fewer than $20 \%$ of individuals with hypertension have well-controlled bloodpressure levels. Investigators of the HOPE 4 programme developed a country-specific model of care that takes into account barriers to effective disease management such as constrained resources. The HOPE 4 cluster-randomized, controlled trial tested this intervention in 1,371 individuals with new or poorly controlled hypertension from urban or rural communities in Malaysia and Colombia. This novel intervention was led by NPHWs and supported by physicians, family and community members. NPHWs were responsible for identifying CVD risk factors and providing antihypertensive drugs and statins (supervised by local physicians), and a friend or family member was involved in the treatment programme to improve drug adherence and health behaviours.

In total, 14 communities (644 participants) were allocated to the intervention and 16 communities (727 participants) to control. At 1 year, the reduction in the Framingham Risk Score for 10 -year CVD risk was $-6.4 \%$ $(95 \% \mathrm{Cl} 8.0$ to -4.8$)$ in the control group and $-11.2 \%(95 \% \mathrm{Cl}-12.9$ to -9.5$)$ in the intervention group, with a difference of change of $-4.8 \%(95 \% \mathrm{Cl}-7.1$ to -2.4 , $P<0.0001)$. The intervention group also had greater absolute reductions in systolic blood pressure, total cholesterol and LDL-cholesterol levels.

To conclude, this tailored model of care reduced the risk of CVD in two middleincome countries through improvements in blood-pressure management. "We believe that a major reason for its success was that our intervention package explicitly targeted barriers identified through extensive health system appraisals," explain the investigators.

Karina Huynh

ORIGINAL ARTICLE Schwalm, J. - -D. et al. A community-based comprehensive intervention to reduce cardiovascular risk in hypertension (HOPE 4): a cluster-randomised controlled trial. Lancet https://doi.org/10.1016/S0140-6736(19)31949-X (2019)

\title{
Clonal haematopoiesis, aortic stenosis and reduced survival after TAVI
}

Clonal haematopoiesis of indeterminate potential (CHIP) is defined as the presence of an expanded somatic blood cell clone without other haematological abnormalities and has been associated with an increased incidence of coronary artery disease and calcification. A new study now shows that CHIP-driver mutations in DNMT3A or TET2 are associated with increased medium-term mortality in patients with severe aortic stenosis undergoing transcatheter aortic valve implantation (TAVI).

The investigators studied 279 patients with severe aortic stenosis and found that $93(33 \%)$ had a CHIP-driver mutation with a variant allele frequency $\geq 2 \%$ in $D N M T 3 A$ $(n=53)$ or TET2 $(n=40)$. The incidence of these mutations increased with age from $25 \%$ in those aged $55-69$ years to $53 \%$ in those aged $90-100$ years.

After a successful TAVI procedure, patients with a DNMT3A or TET2 CHIP-driver mutation had a significantly increased risk of death in the first 8 months. This increased risk of death remained even after accounting for the potential effects of age and sex (HR 3.1, 95\% CI 1.17-8.08, $P=0.022$ ).

With the use of fluorescence-activated cell sorting analysis, patients with a TET2 CHIP-driver mutation were found to have increased levels of circulating non-classical monocytes $\left(\mathrm{CD} 14^{\mathrm{dim}} \mathrm{CD} 16^{++}\right)$, which are known to secrete high levels of inflammatory cytokines. By contrast, patients with a DNMT3A CHIP-driver mutation had a significantly increased $\mathrm{T}_{\mathrm{H}} 17: \mathrm{T}_{\text {reg }}$ ratio, indicating pro-inflammatory $\mathrm{T}$-cell polarization. The researchers speculate that the presence of DNMT3A or TET2 CHIP-driver mutations might have contributed to the development of the aortic stenosis.

Gregory B. Lim

ORIGINAL ARTICLE Mas-Peiro, S. et al. Clonal haematopoiesis in patients with degenerative aortic valve stenosis undergoing transcatheter aortic valve implantation. Eur. Heart J. https:// doi.org/10.1093/eurheartj/ehz591 (2019) RELATED ARTICLES Jaiswal, S. \& Libby, P. Clonal haematopoiesis: connecting ageing and inflammation in cardiovascular disease. Nat. Rev. Cardiol. https://doi.org/10.1038/s41569-019-0247-5 (2019)

\section{RISK FACTORS}

\section{Reductions in LDL-cholesterol and blood- pressure levels have cumulative benefits}

Lifelong genetic exposure to lower levels of both LDL cholesterol (LDL-C) and systolic blood pressure (SBP) is associated with a dose-dependent decreased risk of major coronary events. This finding comes from a new study published in JAMA and presented at the ESC Congress 2019.

The study included data from 438,952 participants enrolled in the UK Biobank. Participants were randomly divided into groups according to genetic LDL-C and SBP scores. The LDL-C score consisted of 100 exome variants previously associated with LDL-C level at the genome-wide level of significance and, similarly, the SBP score consisted of 61 exome variants previously associated with SBP level.

Individuals with an LDL-C genetic score higher than the median had $14.7 \mathrm{mg} / \mathrm{dl}$ lower LDL-C levels and a lower risk of major coronary events (OR 0.73, 95\% Cl 0.70-0.75, $P<0.001$ ) than individuals with an LDL-C genetic score equal to or lower than the median. Similarly, individuals with an SBP genetic score higher than the median had $2.9 \mathrm{mmHg}$ lower SBP levels and a lower risk of major coronary events (OR 0.82, 95\% Cl 0.79-0.85, $P<0.001$ ) than individuals with an SBP genetic score equal to or lower than the median. Of note, individuals with both genetic risk scores higher than the median had $13.9 \mathrm{mg} / \mathrm{dl}$ lower LDL-C levels and $3.1 \mathrm{mmHg}$ lower SBP levels and a log-additive reduction in the risk of major coronary events (OR 0.61, 95\% Cl 0.59-0.64, $P<0.001$ ).

The investigators emphasize that the relationship between long-term exposure to lower LDL-C and SBP levels and the risk of cardiovascular events is independent, additive and dose-dependent, so that even small reductions in LDL-C and/or SBP levels can have clinically meaningful benefits over the course of a lifetime.

Gregory B. Lim

ORIGINAL ARTICLE Ference, B. A. et al. Association of genetic variants related to combined exposure to lower low-density lipoproteins and lower systolic blood pressure with lifetime risk of cardiovascular disease. JAMA https://doi.org/10.1001/ jama.2019.14120 (2019) 